\title{
Retroperitoneal schwannoma: common tumour in an uncommon place
}

\author{
S N Deshmukh ${ }^{1}$, S G Agarwal ${ }^{1}$, S A Bolde', V R Shah ${ }^{3}$ \\ 'Department of Surgery, ${ }^{2}$ Department of Pathology, ${ }^{3}$ Department of Radiology, \\ Dr Vaishampayan Memorial Govt. Medical College, Solapur, Maharashtra, India.
}

Key words: Schwannoma; retroperitoneum

\section{Introduction}

Schwannomas are tumours that originate from Schwann cells. [1] Schwannomas usually arise from sensory nerves, however, motor nerve origin is also reported.[2] They are ordinarily seen in the head, neck and extremities, and are only rarely found in the retroperitoneum. A retroperitoneal location accounts for $0.3 \%$ to $3.2 \%$ of all primary schwannomas. [1] Here we report a case of benign retroperitoneal schwannoma.

\section{Case History:}

An 18-year-old male presented with an abdominal mass of 6 months and abdominal pain of one month. His bowel and bladder habits were normal. Abdominal examination revealed a large, firm, non-tender mass with a smooth surface in the left lumbar region. The mass did not move with respiration. There was no lymphadenopathy. Laboratory parameters were within normal limits. There were no cutaneous stigmata of neurofibromatosis. Abdominal contrast CT scan showed a $23 \times 15 \times 15 \mathrm{~cm}$ lesion with multiple foci of calcification, well defined margins and a peripheral non-enhancing area of necrosis.(Fig 1A) A provisional diagnosis of retroperitoneal sarcoma was made and an exploratory laparotomy was performed. Exploration revealed a large encapsulated retroperitoneal lesion without invasion into surrounding structures. The mass was completely excised [Fig 1B].

Post-operative recovery was uneventful. Histopathological examination revealed a schwannoma (Fig 2), Immunohistochemical staining

Correspondence :

Deshmukh SN

Email : santoshkumardeshmukh@yahoomail.com was positive for S-100 protein. The patient was free from recurrence after 6 months.

\section{Discussion:}

Retroperitoneal schwannomas are rare and usually found in the paravertebral space or presacral region.[3] These tumours most commonly occur between 40 and 60 years of age, with a male to female ratio of 2:3[4]. Patients with retroperitoneal schwannomas most commonly present with abdominal distention and pain. Atypical presentations include flank pain, haematuria, headache, secondary hypertension, recurrent urinary colics and Horner's syndrome [4]. Retroperitoneal schwannomas are solitary and slow-growing so they may remain asymptomatic in the retro-peritoneum until they reach a large size and cause a mass effect or nerve impingement [5] At the time of diagnosis, the size of a retroperitoneal schwannoma is usually more than $8 \mathrm{~cm}$ in diameter. Most schwannomas are benign and malignant degeneration is extremely rare. Malignant degeneration particularly occurs in association with Von Recklinghausen's disease. Retroperitoneal schwannoma should be differentiated from other retroperitoneal tumours like ependymoma, chordoma, chondrosarcoma, giant cell tumour, aneurysmal bony cyst, lymphoma and ganglioneuroma. [4] Radiographic modalities do not differentiate benign from malignant disease unless tumour invasion or metastasis is seen. Definitive diagnosis is based on histopathlogical examination and immunohistochemistry. Microscopically the hallmark of a schwannoma is the pattern of alternating Antoni $\mathrm{A}$ and $\mathrm{B}$ areas. Immunohistochemically schwannomas are characterized by S-100 positivity. [1] Complete surgical excision is the only valid treatment for schwannomas; since, schwannomas are not sensitive to radiotherapy and chemotherapy. [3] Tumour recurrence or malignant transformation after 


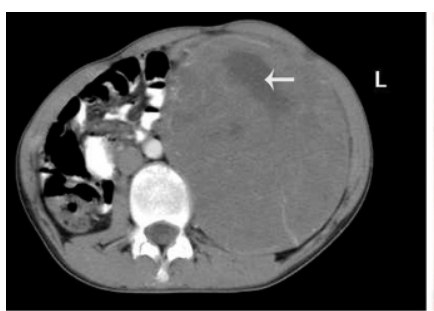

A

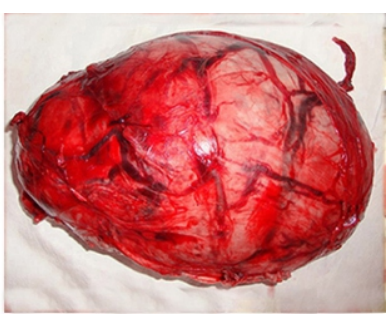

B
Figure 1A. Abdominal contrast CT scan showing large well defined retroperitoneal tumour with peripheral rim enhancement and area of necrosis. (white arrow) ; 1 B. Resected tumour

complete resection is very rare for retroperitoneal schwannoma.[1] There are few reported cases in which metastases occurred after resection of a histologically benign schwannoma.[4]So postoperative monitoring is necessary.

\section{References:}

1. Kudo T, Kawakami H, Kuwatani M, Ehira N, et al. Three cases of retroperitoneal schwannoma diagnosed by EUS-FNA. World J Gastroenterol 2011; 17(29):4359-3464.

2. Choudry HA, Nikfarjam M, Liang JJ, Kimchi ET, et al.(2009) Diagnosis and management of retroperitoneal ancient schwannomas. World J Surg Oncol 2; 7:12.

3. Kapan M, Onder A, Gumus M, Gumus H et al (2011) Retroperitoneal Schwannoma. JSCR 10:1

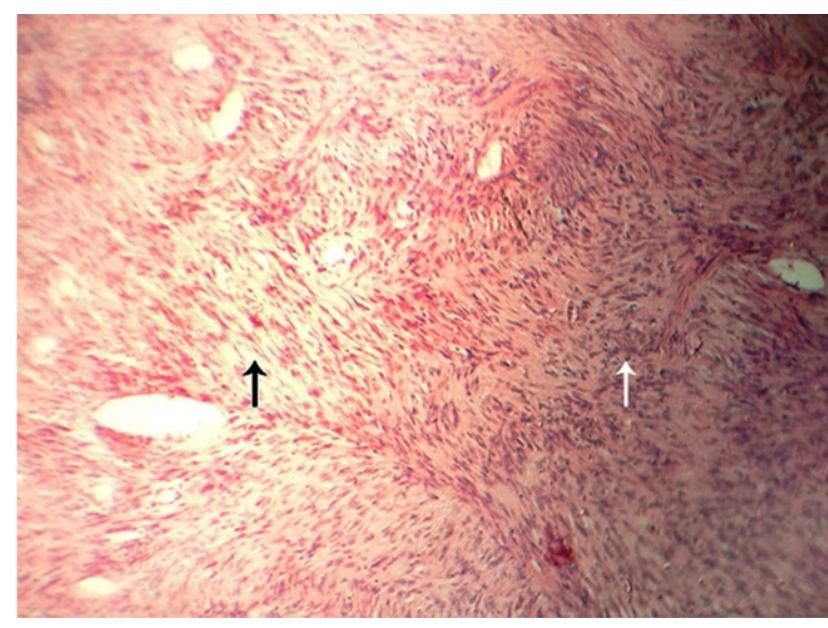

Figure 2. Microphotograph showing, Antoni-A area, hypercellular area showing nuclear palisading (white arrow) and Antoni-B area, Hypocelluar area. ( black arrow) ( Stain. H \& E, 10x )

4 Aslan Y, Aydin AO,Tekdogan UY,Atan A (2010) Retroperitoneal schwannoma: A case report and review of the literature. J Neurol Sci [Turk] 27(3): 353-358.

5 Getachew MM, Whitman GJ, Chew FS (1994) Retroperitoneal schwannoma.Am J Roentgenol 163(6):1356. 